\title{
Adolescents referred following overdose
}

\author{
Support for Hawton's classification and the role of a \\ primary child and adolescent mental health worker
}

\author{
Gillian Davies and Samantha Ames
}

\begin{abstract}
Services for adolescents who overdose remain variable in the UK despite College guidelines. The problem is significant in ferms of the demand placed on services, the level of distress it represents and the high eventual mortality rate. This study was undertaken to gain an understanding of the local picture with a view to improving service provision. The study revealed a high level of psychiatric morbidity, considerable drug and alcohol misuse and paracetamol as the most commonly taken substance. The findings supported Hawton's classification into three distinct groups, confirming it as a simple but useful system. This may provide a means of screening adolescents who overdose to improve service delivery to those most in need within the resources available. The possible role of the primary child and adolescent mental health worker is discussed.
\end{abstract}

Overdose behaviour is a significant problem and follow-up studies of teenagers who take overdoses show that up to $11 \%$ will eventually commit suicide (Spirito et al, 1989). The Royal College of Psychiatrists guidelines (1982) state that admission to hospital is desirable in most cases of parasuicide in children and adolescents, a child psychiatric team should be directly involved and that a response within 48 hours would be reasonable. These guidelines were reviewed at the College meeting in Dublin in 1995, and it was clear that few people were adhering to them with great variation around the country. The meeting concluded that revision of the guidelines was not necessary but they should be restated with the aim of encouraging a more uniform practice throughout the UK.

The aim of our study was to define the characteristics of the adolescents who take overdoses in West Glamorgan and to determine the usefulness of Hawton's classification (Hawton et al, 1982).

\section{The study}

The case notes of 50 consecutively referred adolescents who had taken overdoses in West Glamorgan during April 1995-November 1995 were reviewed. A multiaxial classification (using ICD-10 for axis I) was completed for each subject. Details regarding the substance taken, associated drug and alcohol use, previous or subsequent overdose or self-injury and aftercare arrangements were recorded.

Each case was rated according to Hawton's classification as being either:

(a) Acute (Group I): problems identified at the time of the overdose had persisted for less than one month; no behavioural disturbance.

(b) Chronic (Group II): problems identified at the time of the overdose had persisted for one month or more; no behavioural disturbance.

(c) Chronic with behavioural disturbance (Group III): problems identified at the time of the overdose had persisted for one month or more; recent behavioural disturbance (e.g. repeated truanting, stealing, drug taking, heavy drinking, fighting. in trouble with the police).

We identified the characteristics of each of the three subgroups with respect to diagnosis (axis 1 and 5), associated drug and alcohol misuse, and follow-up offered.

\section{Findings}

During the study period, 157 adolescents presented with overdoses to the three accident and emergency departments in West Glamorgan. Eighty-one were admitted and 50 subsequently referred for psychiatric assessment. Of these, five were from the Dyfed area. The referred sample 
consisted of 47 girls and three boys aged between 11 and 16.

The most frequent diagnosis was adjustment disorder $(38 \%)$, followed by conduct disorder $(28 \%)$ and depressive episode $(20 \%)$. No psychiatric disorder was found in two cases $(4 \%)$. The most common substance taken was paracetamol with $66 \%$ having taken paracetamol alone or in combination with another substance. The reported rate of drug and alcohol misuse was $32 \%$. The repetition rate of previous overdoses was $26 \%$ with a further $10 \%$ repeating during the study period. The rate of previous self-injury was $10 \%$, with $6 \%$ repeating during the study period. Of the $66 \%$ offered follow-up in West Glamorgan. two-thirds attended at least once.

Using Hawton's classification there were 12 (24\%) in Group I, 21 (42\%) in Group II and 17 (34\%) in Group III.

Excluding the cases of accidental ingestion, all in Group 1 had a diagnosis of adjustment disorder with brief depressive reaction and were found to have minimal psychosocial disturbance. They did not misuse alcohol or drugs and 5/12 were offered follow-up of which only three attended.

In Group II, 47.6\% were depressed, and 23.8\% had either a significant adjustment disorder or depressive conduct disorder. The subjects had a relatively high drug and alcohol use, with onethird of the group using either drugs or alcohol. The most frequently used categories on axis $V$ were discordant intrafamilial relationships, anomalous family situation and stresses in school or work environment, the latter not having been evident in the other two groups. All the adolescents were offered follow-up and 13/19 $(68.4 \%)$ attended.

The Group III, 76.5\% had a diagnosis of conduct disorder and the highest rate of drug and alcohol misuse. Four of the six drug users in this group were multiple drug users. This group had the highest incidence of psychosocial disturbance characterised by discordant intrafamilial relationships, inadequate/inconsistent parental control and anomalous family situation. Twelve out of 17 were offered follow-up and eight (66\%) attended.

\section{Comment}

Of the 157 adolescents presenting with overdoses, only 50 were eventually assessed by the child psychiatric service following screening by casualty staff and paediatricians. Despite this screening, the referred group still contained a wide range of adolescent problems ranging from youngsters with minimal mental health problems to those with severe psychiatric disorder.
Our findings support other studies showing that paracetamol is the most frequently used drug in overdose (Hawton et al, 1996) and the rate of drug and alcohol misuse is a significant problem (Hawton et al, 1982; Brent et al, 1988; Spirito et al, 1989; Kerfoot et al, 1996). Our finding of a repetition rate of previous self-harm $(36 \%)$ is comparable to Hawton's study (Hawton et al, 1996), who also reports a rising repetition rate. Attendance at follow-up appointments was higher $(66 \%)$ than the $56 \%$ in Taylor's study (Taylor \& Stansfield, 1984).

Our finding of $20 \%$ of subjects having depressive disorder is similar to that reported by Taylor \& Stansfield (1984) but lower than the 67\% found by Kerfoot and colleagues (1996). The rate of $28 \%$ for conduct disorder is in keeping with other authors (Brent et al, 1988; Trautman et al, 1993).

This study supports the existence of three distinct subgroups as suggested by Hawton. The main intervention required with Group I would seem to be a discussion about alternative problem-solving and advice to the parents about neither over- nor under-reacting to the event. Ongoing psychiatric help from a child psychiatric team does not seem to be indicated, although the young person and their family should know how to avail themselves of this if required.

In Group II, we noted a significant amount of depression in contrast to Hawton's description (Hawton et al, 1982) of adolescents who are "sad but not suffering from a depressive illness in any formal sense". This may be accounted for by the improved recognition of depressive illness in young people, and also the rising incidence (Ryan et al, 1992). It would appear that this is the group where intervention by the child psychiatric team can be most effective, as motivation for treatment is high and appropriate help for depression and other psychiatric disorders can be given.

Group III has the poorest prognosis, and is the most difficult to help. The role of the child psychiatrist with these youngsters is often to work in partnership with social services and the education department to try to establish appropriate levels of care, control and education. This is the group whose drug and alcohol use is likely to continue and to become a problem in its own right.

From these observations, we feel that the following suggestions may be helpful in the commissioning, planning and provision of services for adolescents who take overdoses. First, in accordance with the College guidelines, we agree that it is desirable that most adolescents are admitted to hospital following an overdose. Secondly, all these adolescents should be screened regarding the necessity of referral to the child psychiatric team. In our service, this 
would result in an increased number requiring an initial screen, which could be undertaken by a primary child and adolescent mental health worker as discussed in the recent Health Advisory Service (1995) report. This worker could be a mental health worker working at the interface between tier 1 and tier 2 , in the tiered system of mental health provision, or a member of the multi-disciplinary child psychiatric team undertaking the same duties. In either case discussion could take place and regular supervision given from the tier 2 team.

At ward level, the adolescents in Group 1 could be offered minimal intervention as outlined above and would not need referral to the child psychiatric team whereas those in Group II and III could access more specialist help from the team (tier 2) making use of limited resources. Once established, the role of the mental health worker could broaden consultation and training to staff working on the wards and in casualty departments.

\section{Conclusions}

In view of limited resources and pressure on services it is unlikely that many services including our own will be able to achieve the goals recommended by the College. In West Glamorgan only a third of adolescents presenting following an overdose are referred to the child psychiatric service, meaning that many are failing to receive appropriate assessment and therefore there is a large unmet need. To address this, we believe that commissioners could consider the appointment of a child and adolescent mental health worker to undertake this work and we propose that Hawton's classification could be a useful screening tool for use in this situation.

\section{References}

Brent, D. A., Perper, J., Goldstein, C., et al (1988) Risk factors for adolescent suicide: A comparison of adolescent suicide victims with suicidal inpatients. Archives of General Psychiatry. 45, 581-588.

HAWTON, K., OSBORNE, M., O'GraDY, J., et al (1982) Classification of adolescents who take overdoses. British Journal of Psychiatry. 140, 124-131.

- FAGG, J. \& SimkIN. S. (1996) Deliberate self-poisoning and self-injury in children and adolescents under 16 years of age in Oxford, 1976-1993. British Journal of Psychiatry, 169, 202-208.

HEALTH ADVISORY SERVICE (1995) Thematic Review of Child and Adolescent Mental Health Services. Together We Stand. London: HAS.

KERFOOT, M., DYER, E., HARRINGTON, V., et al (1996) Correlates and short-term course of self-poisoning in adolescents. British Joumal of Psychiatry, 168, 38-42.

ROYAL COLLEGE OF PSYCHIATRISTS (1982) The management of parasuicide in young people under 16. Bulletin of the Royal College of Psychiatrists, 6, 182-185.

RYAN, N. D.. Williamson, D. E.. IYERAGaR, S., et al (1992) A secular increase in child and adolescent onset affective disorder. Journal of the American Academy of Child and Adolescent Psychiatry. 31, 600-605.

SPIRTO, S., BROWN, L., OVERHOLSER, J., et al (1989) Attempted suicide in adolescence: a review and critique of the literature. Clinical Psychology Review. 99. 335-363.

TAYLOR. E. A. \& Stansfield, S. A. (1984). Children who poison themselves. A clinical comparison with psychiatric controls. British Joumal of Psychiatry. 145. 127-135.

Trautman, P. D., Stewart, M. D. \& Morishma, A. (1993) Are adolescent suicide attempters noncompliant with outpatient care? Joumal of the American Academy of Child and Adolescent Psychiatry, 32, 89-94.

Gillian Davies, Consultant Child and Adolescent Psychiatrist, Harvey Jones Unit, Whitchurch, Cardiff, and *Samantha Ames, Senior Registrar in Child and Adolescent Psychiatry. 'Trehafod' Child and Family Clinic, Waunarlwydd Road, Sketty, Swansea SA2 OGB

*Correspondence 